\title{
A fast-growing cold skin abscess revealing disseminated Mycobacterium intracellulare infection in an HIV-infected patient
}

International Journal of STD \& AIDS $0(0)$ 1-3

(C) The Author(s) 2017

Reprints and permissions:

sagepub.co.uk/journalsPermissions.nav DOI: 10.1 I77/09564624I7748240 journals.sagepub.com/home/std (SAGE

\author{
Pedro Mendes-Bastos ${ }^{1,2}$, Susana Brás' and Rodrigo Carvalho',2
}

\begin{abstract}
A 66-year-old woman with HIV-I infection recently commenced on antiretroviral therapy (CD4+25 cells $/ \mathrm{mm}^{3}$ was referred to the Dermatology Clinic the following month due to a well-demarcated nodule in the extensor surface of the left arm with evident fluctuation but only slight pain on palpation, with no increase in temperature. Surgical drainage was performed with aspiration of yellowish-green exudate, with no characteristic smell. In culture of cutaneous exudate, Mycobacterium intracellulare was isolated. Upon careful review of the laboratory tests that were in progress at discharge, the same agent was isolated in one of the bronchoalveolar lavage cultures. The diagnosis of cutaneous abscess caused by M. intracellulare from hematogenous dissemination of lung infection was made. The patient was treated with clarithromycin, ethambutol and rifabutin for 24 months. M. intracellulare species and Mycobacterium avium constitute the Mycobacterium avium-intracellulare complex (MAC), responsible for the majority of human infections by atypical mycobacteria. They are ubiquitous bacteria and MAC infection mainly affect immunocompromised patients, with $M$. intracellulare being isolated in $<5 \%$ of HIV patients with MAC infection. Cutaneous infection is rare and may present clinically with erythematous plaques, chronic ulcers or abscesses. When present, skin involvement is usually secondary to pulmonary infection.
\end{abstract}

\section{Keywords}

Mycobacterium avium-intracellulare infection, infectious skin diseases, human immunodeficiency virus

Date received: 20 August 2017; accepted: 21 November 2017

\section{Case report}

A 66-year-old female patient originally from GuineaBissau was referred to the Dermatology Clinic due to a fast-growing asymptomatic skin nodule on the left arm, present for one week. Two months before, she had been admitted to the Infectious Diseases Ward for Pneumocystis jiroveci pneumonia and Cytomegalovirus proctitis, revealing an undiagnosed human immunodeficiency virus-1 (HIV-1) infection (CD4+ cell count of 25 cells $/ \mathrm{mm}^{3}$, viral load of 507,653 copies $/ \mathrm{mL}$ ). After appropriate medical treatment and the start of tenofovir/emtricitabine and raltegravir (TDF/FTC + RAL), the patient became asymptomatic and was discharged with residual lung disease on CT scan.

On skin examination, a single well-demarcated and non-ulcerated $4-\mathrm{cm}$ diameter skin nodule on the extensor surface of the left arm was observed (Figure 1). There was fluctuation upon palpation but no pain, warmness or redness (Figure 2). No lymphadenopathy was present and the patient denied any local trauma. This skin abscess was surgically drained, releasing an abundant odorless yellowish thick fluid that was fully aspirated and sent for culture - aerobic and anaerobic bacteria, fungi and mycobacteria. The

\footnotetext{
'Department of Dermatology and Venereology, Centro Hospitalar de Lisboa Central, Lisboa, Portugal

${ }^{2}$ Dermatology Centre, Hospital CUF Descobertas, Lisboa, Portugal
}

\section{Corresponding author:}

Pedro Mendes-Bastos, Dermatology Centre, Hospital CUF Descobertas, Rua Mário Botas, Lisboa 1998-018, Portugal.

Email: pmendesbastos@gmail.com 


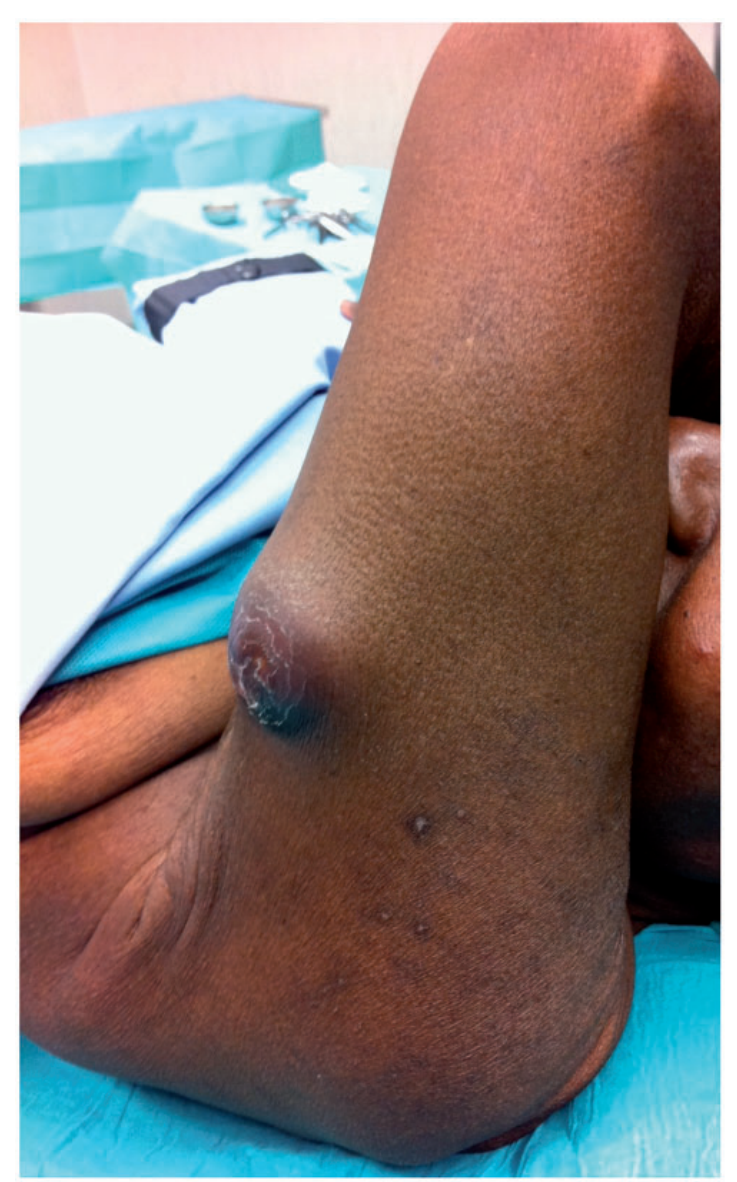

Figure I. A single well-demarcated and non-ulcerated 4-cm diameter skin nodule on the extensor surface of the left.

cystic cavity was repeatedly injected with a diluted solution of povidone-iodine. Direct examination of the skin exudate was negative, including for acid-fast bacilli. Two weeks later, the skin nodule relapsed and the procedure was repeated.

One month after the first surgical drainage, Mycobacterium intracellulare was identified as the causative agent of the skin abscess (culture and nuclear hybridization). At that time, the same agent was isolated from one sample of the bronchoalveolar lavage collected three months before as an inpatient.

We made the diagnosis of a disseminated $M$. intracellulare infection. The patient was kept on $\mathrm{TDF} / \mathrm{FTC}+\mathrm{RAL}$ in addition to clarithromycin $250 \mathrm{mg}$ bid, ethambutol $1200 \mathrm{mg}$ qd and rifabutin $300 \mathrm{mg}$ qd for 24 months.

The skin nodule regressed after two weeks and computed tomography of the thorax showed no residual lung disease at nine months of therapy. After 24 months of therapy, the immune status had improved $\left(\mathrm{CD} 4+\right.$ cell count of 261 cells $/ \mathrm{mm}^{3}$, viral load $<20$

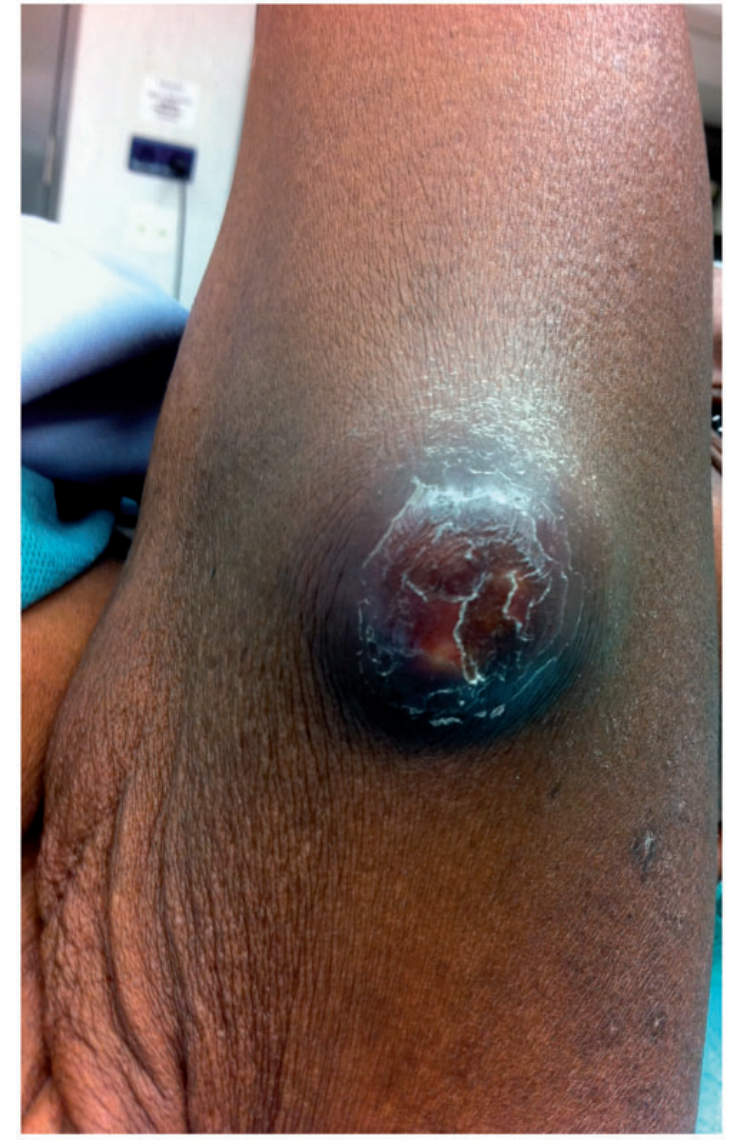

Figure 2. Fluctuation upon palpation was evident, with no pain, warmth or redness.

copies $/ \mathrm{mL}$ ) and the antimycobacterial therapy was stopped. No lung or skin recurrence occurred at six months of follow-up.

\section{Discussion}

A global decrease in tuberculosis was accompanied by an increased awareness of atypical mycobacteria (AM) -associated disease. ${ }^{1}$ Due to similar clinical, etiological and antigenic similarities, some groups of AM are considered together as complexes: Mycobacterium avium and $M$. intracellulare are grouped together as the Mycobacterium avium-intracellulare complex (MAC). The MAC complex agents are ubiquitous slowgrowing pathogens and environmental exposure is considered the predominant pathway to disease. Regarding cutaneous AM disease occurring globally, several recent case series classify $65-86 \%$ of the affected patients as immunocompetent. ${ }^{2-4}$ MAC disease, on the other hand, is much more common in immunosuppressed individuals, mainly in persons with advanced HIV infection, and the more frequent clinical presentation is lung disease or disseminated disease. ${ }^{1}$ 
Disseminated AM disease in HIV patients is due to MAC in $>90 \%$ of cases, with more than $90 \%$ of those infections due to $M$. avium; it tends to occur in patients who are severely immunocompromised, as evidenced by very low CD4+ T-cell counts. ${ }^{5}$ Skin involvement revealing MAC disseminated disease has been rarely reported, both in healthy and in immunocompromised patients ${ }^{6,7}$ and is not considered a typical clinical presentation. ${ }^{1}$ According to most studies, the importance of MAC in cutaneous AM disease is limited. As most cases of cutaneous AM infection occur in immunocompetent patients and are limited to skin and soft tissues, other agents as Mycobacterium marinum or Mycobacterium fortuitum are much more commonly reported. ${ }^{1,4,8}$ Primary cutaneous infection by MAC is rare, and excluding concomitant pulmonary disease is considered mandatory, ${ }^{9}$ especially in the context of immunosuppression. The variety of clinical manifestations is wide and non-specific, including erythematous plaques, ulcers and abscesses. ${ }^{1,3,6,7,10}$ As a crucial part of multidisciplinary teams, the dermatologist is on the front line of care for severely immunocompromised patients and a high level of suspicion is mandatory when approaching these cases.

\section{Declaration of conflicting interests}

The author(s) declared no potential conflicts of interest with respect to the research, authorship, and/or publication of this article.

\section{Funding}

The author(s) received no financial support for the research, authorship, and/or publication of this article.

\section{References}

1. Griffith D, Aksamit T, Brown-Elliot B, et al. An official ATS/IDSA statement: diagnosis, treatment and prevention of nontuberculous mycobacterial diseases. Am J Respir Crit Care Med 2007; 175: 367-416.

2. Song H, Lee H, Choi G, et al. Cutaneous nontuberculous mycobacterial infection: a clinicopathological study of 7 cases. Am J Dermatopathol 2009; 31: 227-231.

3. Lee W, Kang S, Sung H, et al. Non-tuberculous mycobacterial infections of the skin: a retrospective study of 29 cases. J Dermatol 2010; 37: 965-972.

4. MAE-K. Atypical mycobacterial cutaneous infections in Egyptians: a clinicopathological study. J Dermatol 2014; 41: $303-310$.

5. Horsburgh $\mathrm{C} \mathrm{Jr}$, Gettings $\mathrm{J}$, Alexander $\mathrm{L}$, et al. Disseminated Mycobacterium avium complex disease among patients infected with human immunodeficiency virus, 1985-2000. Clin Infect Dis 2001; 33: 1938-1943.

6. Hide M, Hondo $\mathrm{T}$, Yonehara $\mathrm{S}$, et al. Infection with Mycobacterium avium-intracellulare with abscess, ulceration and fistula formation. Br J Dermatol 1997; 136: $121-123$.

7. Colucci R, Agnoletti A, Arunachalam M, et al. Skin ulcer as presenting manifestation of pulmonary Mycobacterium avium infection. Int J Dermatol 2014; 53: e192-e193.

8. Lamb R and Dawn G. Cutaneous non-tuberculous mycobacterial infections. Int $J$ Dermatol 2014; 53: 1197-1204.

9. Glassroth J. Pulmonary disease due to non-tuberculous mycobacteria. Chest 2008; 133: 243-251.

10. Piersimoni $\mathrm{C}$ and Scarparo C. Extrapulmonary infections associated with non-tuberculous mycobacteria in immunocompetent persons. Emerg Infect Dis 2009; 15: 1351-1357. 